\title{
Paget's disease of the penis and scrotum mimicking cutaneous metastatic lesion from gastrointestinal or genitourinary tract adenocarcinoma
}

Tharveen Nair ${ }^{1,2}$, Tan Jih Huei ${ }^{1,2}$, Henry Tan Chor Lip ${ }^{1,2}$, Durvesh Lachman Jethwani ${ }^{1}$, Ooi Chin Chuan, Shamsuddin Omar ${ }^{1}$

${ }^{1}$ Hospital Sultanah Aminah, Johor Bahru, Malaysia

${ }^{2}$ National University of Malaysia

Keywords: Extramammary Paget's disease; cutaneous metastasis of adenocarcinoma; penoscrotal

\section{Introduction}

Extramammary Paget's disease (EMPD) is an uncommon, slow-growing intra-epithelial adenocarcinoma. It normally occurs at areas rich with apocrine glands of the perineum, vulva, axilla and male genitalia including penis and scrotum.

EMPD of the penis and scrotum was first reported by Crocker Radcliffe in 1889. It is predominantly common amongst elderly individuals but due to its rarity, the incidences are relatively unknown [1]. These lesions are usually presented as non-specific erythematous, eczematous or circular lesions with pruritus and pain.

Here, we present a primary EMPD patient with nodular growth in penis and scrotum, having the background of erythematous lesion of the left penoscrotal region. The initial biopsy suggested an adenocarcinoma of the uncertain primary which warranted further investigations before definitive surgical resection of EMPD.

In this case report, we intend to highlight the importance of early referral for a biopsy and the challenges of management.

\section{Case presentation}

A 70-year-old male, presented with an erythematous skin lesion and painless mass over left scrotum and penis for 2 months. He had no history of weight loss or a family history of malignancy. The clinical examination revealed a $4 \mathrm{~cm} \times 5 \mathrm{~cm}$ fungating, exophytic, nodular, yellowish with areas of necrotic patches at the left penoscrotal junction.

The lesion extended to the left foreskin which was reddish and nodular. There was erythematous skin involvement over the left hemi-scrotum (Figure 1). There was no significant palpable inguinal lymphadenopathy and no testicular mass, pleural effusion or abdominal mass.

Correspondence: Tan Jih Huei

E-mail: huei87@hotmail.com

Received: 03-01-2020 Accepted: 13-04-2020

(iD) https://orcid.org/0000-0001-9833-5164

DOI: http://doi.org/10.4038/sljs.v38i1.8660

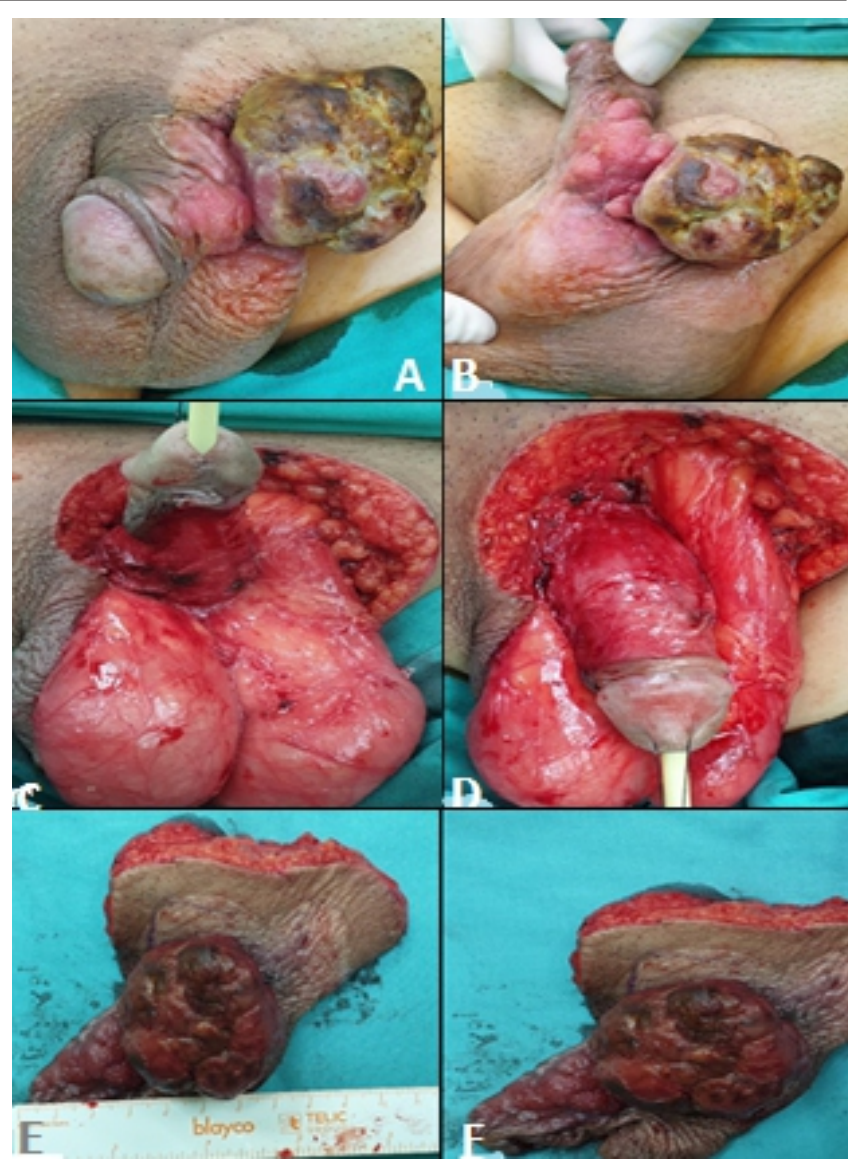

Figure 1A \& 1B. Anterior and left lateral aspect of the penoscrotal lesion where there is a fungating, exophytic, nodular, and yellowish with area of necrotic patches with extension of nodular reddish lesion to the left foreskin. There is also erythematous skin involvement of the left hemiscrotum.

Figure 1C, 1D. Appearance of the genital region after the wide local en-bloc excision.

Figure 1E, 1F. The wide local excision specimen of the left penoscrotal lesion measuring $4 \mathrm{~cm}$ in length and $5 \mathrm{~cm}$ in height.

The biopsy from the penile and scrotal lesion revealed, adenocarcinoma of unknown origin with possible primaries of gastrointestinal, genitourinary tract or primary skin adnexal. The atypical cells proliferation within the epidermis and in areas at the dermo-epidermal junction with increased mitosis. The tumour cells displayed moderate pleomorphism 


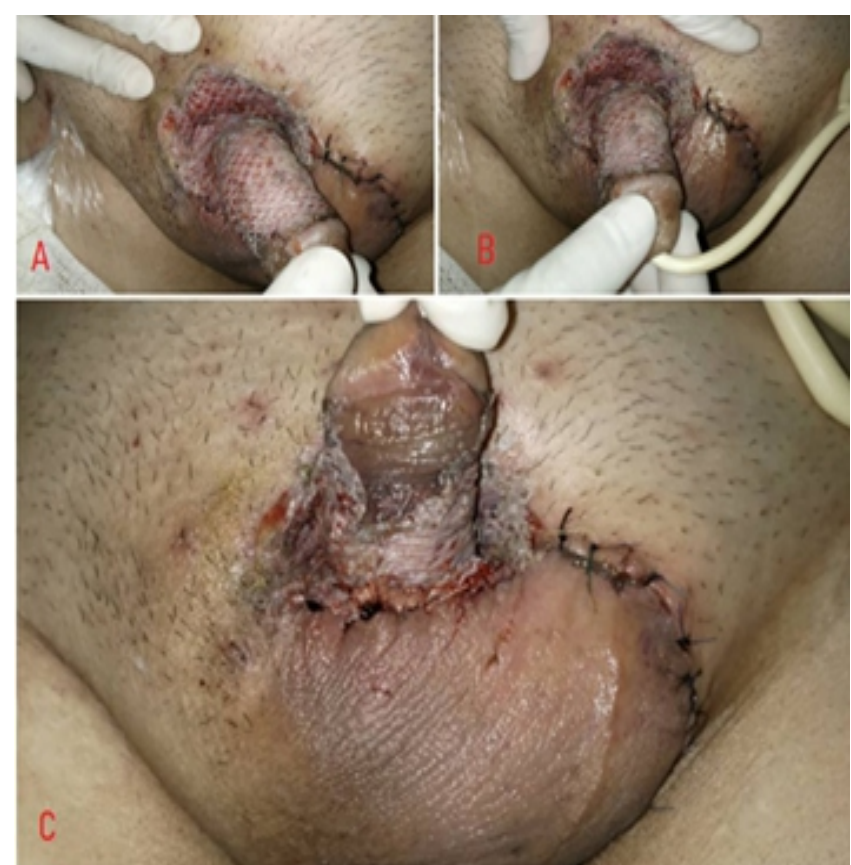

Figure 2 A, B, C. Post-operative picture at day 5 revealed good graft take for the scrotal advancement flap and split skin grafting.

and abundant pale cytoplasm. The immunohistochemistry stain revealed mucin positive, CK7, EMA and CEA positive, but negative for CK 20 .

The contrast-enhanced computed tomography (CECT) of the neck, thorax, abdomen and pelvis revealed no primary lesions or visceral metastasis while the cystoscopic examination revealed no abnormality in the bladder and urethra.

Wide local excision of the left penoscrotal lesion was performed with scrotal advancement flap and split-thickness graft (SSG) (Figure 1). The skin graft healed on subsequent follow up (Figure 2).

The final histopathological report presented, ulcerated surface with areas of malignant epithelial cells involving the dermis with the presence of lymphovascular invasion and skin adnexa. The intracytoplasmic mucin was PAS-positive.

The malignant cells were tested positive for CK7, CEA and focally positive for GCDFP, p63 and p40 and negative for CK20, CDX2 and PSA. The adjacent epidermis showed intraepidermal Paget's disease with prominent nucleoli and pale eosinophilic cytoplasm with clear margins

\section{Discussion}

Extramammary Paget's disease usually occurs in the age group of 60-80 years old with the highest incidence at the age of 65 years. The current case fits the age profile similar to other reported publications of cutaneous metastatic adenocarcinoma of unknown primary [2].
EMPD can be either primary or secondary. Primary EMPD is the common form where an intraepithelial neoplasm originates within the epidermis and apocrine glands. It is not associated with a distant malignancy.

However, it progresses slowly to an invasive or metastatic tumour over time [1]. On the other hand, secondary EMPD develops from the epidermotropic spread of malignant cells from an underlying adenocarcinoma from the dermal adnexal glands or nearby organs to the lesions usually the genitourinary or gastrointestinal tract [3].

Although the secondary EMPD in the penoscrotal region is low, most often it arises from genitourinary [4].

This is excluded in our case by cystoscopy examination.

The final histopathological report revealed the lesion stained positive for CK7 and negative for CK20 which concludes that this is a primary EMPD. Usually, secondary EMPD would stain positive for CK7 and CK20 but CK20 positivity is not specific for secondary EMPD [3, 4].

Usually, primary lesions of EMPD presents in a non-specific manner. Its typical presentation is a well-demarcated, erythematous plague lesion with additional features of ulceration or bleeding.

Patients can be completely asymptomatic or may have pruritus, pain or burning [1]. When the perineum lesion is associated with nodular growth, it may mimic cutaneous metastasis from adenocarcinoma of the genitourinary tract or gastrointestinal system [2].

This difficult clinical differentiation was observed in this current case. Hence, appropriate cystoscopy and contrasted computed tomography scan were performed.

Other lesions that may mimic EMPD include various dermatitides. This has caused the delay of diagnosis with a median to the diagnosis of 2 years from the onset of symptoms [5]. Such delay may influence the survival as $10 \%$ of primary EMPD may have nodal involvement which had worse survival of 0 to $24 \%$ at 5 years [1]. As up to $30 \%$ of EMPD associated with an underlying malignancy, identify this telltale sign early may potentially cure the underlying adenocarcinoma before it is advanced.

The gold standard treatment of EMPD is wide local excision (WLE). The dilemma faced by many surgeons after WLE is the high recurrence rates which can range from $31 \%$ to $61 \%$. The reason behind this is because EMPD usually presents as a multifocal growth with subclinical extension into normalappearing skin. As a result, the surgical resection margins may be compromised and leading to a higher recurrence rate. 
Accepted surgical margin recommendations are about 1 to $5 \mathrm{~cm}$ which is acceptable in this patient as the deep margin is $1.3 \mathrm{~cm}$ away from the malignant cells. To overcome the difficulty in obtaining a clear surgical margin, techniques like frozen section analysis and Mohs micrographic surgery (MMS) have been employed.

For frozen section, results are contradicting where studies have shown that there is no association between margin status and recurrence rates. MMS seems to offer the ultimate margin control where it can observe nearly $100 \%$ of the margin [1].

\section{Conclusion}

EMPD is a rare disease which can be primary or secondary within a wide range of malignancy. When there is atypical growth, it may mimic a cutaneous metastatic lesion from gastrointestinal or genitourinary adenocarcinoma. Early referral for biopsy and workup to exclude secondary EMPD or cutaneous metastasis are essential before advancing to visceral malignancy.
All authors disclose no conflict of interest. The study was conducted in accordance with the ethical standards of the relevant institutional or national ethics committee and the Helsinki Declaration of 1975, as revised in 2000 .

\section{References}

1. Simonds RM, Segal RJ, Sharma A. Extramammary Paget's disease: a review of the literature. Int J Dermatol. 2019 Aug;58(8):871-9. https://doi.org/10.1111/ijd.14328

2. Junqueira ALA, Corbett AMF, de Oliveira Filho J, Nasser K da R, Haddad NN, Tebet ACF. Cutaneous metastasis from gastrointestinal adenocarcinoma of unknown primary origin. An Bras Dermatol. 2015;90(4):564-6.

https://doi.org/10.1590/abd1806-4841.20153175

3. Perrotto J, Abbott JJ, Ceilley RI, Ahmed I. The role of immunohistochemistry in discriminating primary from secondary extramammary Paget disease. Am J Dermatopathol. 2010 Apr;32(2):137-43. https://doi.org/10.1097/DAD.0b013e3181b71481

4. Ekwueme KC, Zakhour HD, Parr NJ. Extramammary Paget's disease of the penis: a case report and review of the literature. J Med Case Reports. 2009 Jan 6;3:4. https://doi.org/10.1186/1752-1947-3-4

5. Isrow D, Oregel KZ, Cortes J, Gomez H, Milikowski C, Feun L, et al. Advanced Extramammary Paget's Disease of the Groin, Penis, and Scrotum. Clin Med Insights Oncol. 2014 Jul 6;8:87-90. https://doi.org/10.4137/CMO.S13107

\section{Learning Points:}

- Extramammary Paget's disease may present with erythematous, eczematous skin lesion at the penoscrotal region.

- Any non-healing lesion in this region should warrant early urology referral for biopsy.

- Clinical assessment with appropriate imaging workups such as axial computed tomography or endoscopic examination is essential to exclude primary malignancy from the genitourinary system or anorectum.

- Complete surgical excision with a clear margin and appropriate skin cover with a local flap may achieve cure. 